\title{
ON THE CONVERSE TO THE ITERATED \\ LOGARITHM LAW
}

BY

C. C. HEYDE

Reprinted from the

JOURNAL OF APPLIED PROBABILITY

Vol. 5, No. 1, pp. 210-215

April 1968

R. Maller et al. (eds.), Selected Works of C.C. Heyde, Selected Works in Probability and Statistics, 


\section{ON THE CONVERSE TO THE ITERATED LOGARITHM LAW}

C. C. HEYDE, University of Sheffield

\section{Introduction}

Let $X_{i}, i=1,2,3, \cdots$ be a sequence of independent and identically distributed random variables with law $\mathscr{L}(X)$ and write $S_{n}=\sum_{i=1}^{n} X_{i}$. If $E X=0$ and $E X^{2}=\sigma^{2}<\infty$, the law of the iterated logarithm (Hartman and Wintner [1]) tells us that

$$
\operatorname{Pr}\left(\limsup _{n \rightarrow \infty}(2 \sigma n \log \log n)^{-\frac{1}{2}}\left|S_{n}\right|=1\right)=1 .
$$

Furthermore, it has recently been shown by Strassen [4] that if

$$
\operatorname{Pr}\left(\limsup _{n \rightarrow \infty}(n \log \log n)^{-\frac{1}{2}}\left|S_{n}\right|<\infty\right)=1,
$$

then $E X=0, E X^{2}<\infty$. The proof of Strassen, however, utilizes deep results of Skorokhod involving the use of an associated Wiener process and it is the object of the present paper to develop a direct approach to this result. As a byproduct, it will be clear how the results of Stone [3] can be extended.

\section{Results}

Theorem. If $E X^{2}=\infty$, then

$$
\operatorname{Pr}\left(\limsup _{n \rightarrow \infty}(n \log \log n)^{-\frac{1}{2}}\left|S_{n}\right|=\infty\right)=1 \text {. }
$$

Proof. Firstly suppose that $\sum \operatorname{Pr}\left[|X|>(n \log \log n)^{\frac{1}{2}}\right]=\infty$. Then, for $N>0$,

$$
\begin{aligned}
\frac{\left|X_{n}\right|}{N[n \log \log n]^{\frac{1}{2}}} & =\frac{\left|S_{n}-S_{n-1}\right|}{N[n \log \log n]^{\frac{1}{2}}} \leqq \frac{\left|S_{n}\right|}{N[n \log \log n]^{\frac{1}{2}}}+\frac{\left|S_{n-1}\right|}{N[n \log \log n]^{\frac{1}{2}}} \\
& \leqq \frac{\left|S_{n}\right|}{N[n \log \log n]^{\frac{1}{2}}}+\frac{\left|S_{n-1}\right|}{N[(n-1) \log \log (n-1)]^{\frac{1}{2}}},
\end{aligned}
$$

so that if $\operatorname{Pr}\left(\left|S_{n}\right|>N(n \log \log n)^{\frac{1}{2}}\right.$ i.o. $)=0$, then we must have

$$
\operatorname{Pr}\left(\left|X_{n}\right|>2 N(n \log \log n)^{\frac{1}{2}} \text { i.o. }\right)=0
$$

Received 25 April 1967. 
and hence, from the Borel-Cantelli Lemma (the $X_{i}$ 's being independent), $\sum \operatorname{Pr}\left[|X|>2 N(n \log \log n)^{\frac{1}{2}}\right]<\infty$. ("i.o." means infinitely often). This clearly contradicts the condition $\sum \operatorname{Pr}\left[|X|>(n \log \log n)^{\frac{1}{2}}\right]=\infty$ so, by appeal to the zero-one law, $\operatorname{Pr}\left(\left|S_{n}\right|>N(n \log \log n)^{\frac{1}{2}}\right.$ i.o. $)=1$. Since $N$ is arbitrarily large, the result of the theorem follows. Consequently, it remains to consider the case where $\sum \operatorname{Pr}\left[|X|>(n \log \log n)^{\frac{1}{2}}\right]<\infty$ but $\sum \operatorname{Pr}\left(|X|>n^{\frac{1}{2}}\right)=\infty$, this last condition being equivalent to $E X^{2}=\infty$. We note, in particular, that $\sum \operatorname{Pr}\left(|X|>n^{1 /(2-\delta)}\right)<\infty$; that is, $E|X|^{2-\delta}<\infty$, every $\delta>0$. Furthermore, if $E X=\mu \neq 0$, the truth of the theorem is evident from the strong law of large numbers so we may subsequently restrict ourselves to the case $E X=0$. We shall proceed by developing a modification of the argument of Stone [3].

Write $F$ for the distribution function of $X$. Also, for a positive constant $K$, let $H$ be the distribution function which results from truncating $X$ at $-K,+K$ and then centering to make the mean zero. Then, there are positive constants $a$ and $b(a+b=1)$ such that $F=a G+b H, G$ being a distribution function. Of course, $b=\operatorname{Pr}(|X| \leqq K)$. Let $Y_{1}, Y_{2}, \cdots$ be independent random variables with common distribution function $G ; Z_{1}, Z_{2}, \cdots$ be independent random variables with common distribution function $H$ and write $T_{n}=\sum_{i=1}^{n} Y_{i}, U_{n}=\sum_{i=1}^{n} Z_{i}$. Also, $\xi_{1}, \xi_{2}, \cdots$ are independent and identically distributed such that $\operatorname{Pr}\left(\xi_{k}=1\right)=a$ and $\operatorname{Pr}\left(\xi_{k}=0\right)=b$. The $Y_{k}$ 's, $Z_{k}$ 's and $\xi_{k}$ 's are taken as mutually independent. We write also, $j(n)=\sum_{i=1}^{n} \xi_{i}, k(n)=n-j(n)$ and $V_{n}=T_{j(n)}+U_{k(n)}$. Clearly $V_{n}$ has the same probabilistic structure as $S_{n}$, so in order to obtain the desired result we have just to show that for any positive $N$,

$$
\operatorname{Pr}\left[(n \log \log n)^{-\frac{1}{2}}\left|V_{n}\right| \geqq N \text { i.o. }\right]=1 .
$$

Now, since $E X=0$ and $E Z_{i}=0$, we have $E Y_{i}=0$ and consequently $\operatorname{Pr}\left(\left|T_{n}\right| \leqq \varepsilon(n \log \log n)^{\frac{1}{2}}\right.$ i.o. $)=1$ for any fixed $\varepsilon>0$. Define events $A_{n}, n \geqq 3$, by

$$
A_{n}=\left\{j(n) \leqq \frac{1}{2}(a+1) n ;\left|T_{j(n)}\right| \leqq \varepsilon(n \log \log n)^{\frac{1}{2}}\right\} .
$$

Then, $\operatorname{Pr}\left(A_{n}\right.$ i.o. $)=1$, for $n^{-1} j(n) \stackrel{\text { a.s. }}{\rightarrow} a$ as $n \rightarrow \infty$ and consequently,

$$
\operatorname{Pr}\left(j(n)>\frac{1}{2}(a+1) n ;\left|T_{j(n)}\right| \leqq \varepsilon(n \log \log n)^{\frac{1}{2}} \text { i.o. }\right)=0 .
$$

("a.s." denotes almost sure convergence). Furthermore,

$$
\begin{aligned}
\operatorname{Pr}\left[(n \log \log n)^{-\frac{1}{2}}\left|V_{n}\right|\right. & \geqq N \text { i.o. }] \\
& =\operatorname{Pr}\left(\left|T_{j(n)}+U_{k(n)}\right| \geqq N(n \log \log n)^{\frac{1}{2}} \text { i.o. }\right) \\
& =\operatorname{Pr}\left(A_{n} ;\left|T_{j(n)}+U_{k(n)}\right| \geqq N(n \log \log n)^{\frac{1}{2}} \text { i.o. }\right) \\
& \geqq \operatorname{Pr}\left(A_{n} ;\left|U_{k(n)}\right| \geqq(N+\varepsilon)(n \log \log n)^{\frac{1}{2}} \text { i.o. }\right) .
\end{aligned}
$$

Also, when $A_{n}$ holds, $k(n) \geqq \frac{1}{2} n b$, and given a small positive $\eta$, we have $(1+\eta) \log \log k(n)>\log \log n$ for $n$ sufficiently large. Thus, 


$$
\begin{aligned}
\operatorname{Pr}\left(\left|V_{n}\right|\right. & \left.\geqq N(n \log \log n)^{\frac{1}{2}} \text { i.o. }\right) \\
& \geqq \operatorname{Pr}\left(A_{n} ;\left|U_{k(n)}\right| \geqq(N+\varepsilon)\left[(1+\eta) 2 b^{-1} k(n) \log \log k(n)\right]^{\frac{1}{2}} \text { i.o. }\right),
\end{aligned}
$$

and the problem is to show that this right right hand probability is unity.

Let $\sigma^{2}=\operatorname{var} Z_{i}=E Z_{i}^{2}$. Then, using the law of the iterated logarithm,

$$
\operatorname{Pr}\left(\left|U_{k(n)}\right| \geqq(N+\varepsilon)\left[(1+\eta) 2 b^{-1} k(n) \log \log k(n)\right]^{\frac{1}{2}} \text { i.o. }\right)=1
$$

provided $(N+\varepsilon)^{2}(1+\eta)<\sigma b$. In order to proceed further, we show that ( $n \log \log n)^{-\frac{1}{2}} T_{n} \stackrel{P}{\rightarrow} 0$ as $n \rightarrow \infty$. (" $P$ " denotes convergence in probability).

Firstly, we recall that $\sum \operatorname{Pr}\left[|X|>(n \log \log n)^{\frac{1}{2}}\right]<\infty$, and consequently $n \operatorname{Pr}\left[|X|>(n \log \log n)^{\frac{1}{2}}\right] \rightarrow 0$ as $n \rightarrow \infty$. Thus, using the degenerate convergence criterion (Loève [2], 317), we shall have $(n \log \log n)^{-\frac{1}{2}} S_{n} \stackrel{P}{\rightarrow} 0$ provided we can show that

(i) $\quad(\log \log n)^{-1} \int_{|x| \leqq(n \log \log n)^{\frac{1}{2}}} x^{2} d F(x) \rightarrow 0 \quad$ as $n \rightarrow \infty$, and

(ii) $\quad n^{\frac{1}{2}}(\log \log n)^{-\frac{1}{2}} \int_{|x| \leqq(n \log \log n)^{\frac{1}{2}}} x d F(x) \rightarrow 0 \quad$ as $n \rightarrow \infty$.

For $n \geqq 3$ we have, using integration by parts,

$$
\begin{aligned}
\int_{|x| \leqq}(n \log \log n)^{\frac{1}{2}} & x^{2} d F(x)=-\int_{0}^{(n \log \log n)^{\frac{1}{2}}} x^{2} d \operatorname{Pr}(|X|>x) \\
\leqq & 2 \int_{0}^{(n \log \log n)^{\frac{1}{2}}} x \operatorname{Pr}(|X|>x) d x \\
= & 2 \int_{0}^{(3 \log \log 3)^{\frac{1}{2}}} x \operatorname{Pr}(|X|>x) d x \\
& +2 \sum_{k=3}^{n-1} \int_{[k \log \log k]^{\frac{1}{2}}}^{[(k+1) \log \log (k+1)]_{\frac{1}{2}}^{\frac{1}{2}}} x \operatorname{Pr}(|X|>x) d x \\
\leqq & \int_{0}^{(3 \log \log 3)^{\frac{1}{2}}} x \operatorname{Pr}(|X|>x) d x \\
& +\sum_{k=3}^{n-1} \operatorname{Pr}\left[|X|>(k \log \log k)^{\frac{1}{2}}\right][(k+1) \log \log (k+1)-k \log \log k] \\
\leqq & \int_{0}^{(3 \log \log 3) \frac{1}{2}} x \operatorname{Pr}(|X|>x) d x+C \sum_{k=3}^{n-1} \log \log k \operatorname{Pr}\left[|X|>(k \log \log k)^{\frac{1}{2}}\right]
\end{aligned}
$$

for some positive constant $C$. Consequently, since $\sum \operatorname{Pr}\left[|X|>(n \log \log n)^{\frac{1}{2}}\right)<\infty$, we have from the Kronecker Lemma (e.g. Loève [2], 238) that 


$$
(\log \log n)^{-1} \int_{|x| \leqq(n \log \log n)^{\frac{1}{2}}} x^{2} d F(x) \rightarrow 0 \quad \text { as } n \rightarrow \infty,
$$

and (i) is established.

In the case of (ii) we have, since $E X=0$,

$$
\begin{aligned}
& \left|\int_{|x| \leqq(n \log \log n)^{\frac{1}{2}}}\right| x d F(x)=\left|\int_{|x|>(n \log \log n)^{\frac{1}{2}}} x d F(x)\right| \\
& \leqq \int_{|x|>(n \log \log n)^{\frac{1}{2}}}|x| d F(x) \\
& =(n \log \log n)^{\frac{1}{2}} \operatorname{Pr}\left[|X|>(n \log \log n)^{\frac{1}{2}}\right]+\int_{(n \log \log n)^{\frac{1}{2}}}^{\infty} \operatorname{Pr}(|X|>x) d x \\
& =(n \log \log n)^{\frac{1}{2}} \operatorname{Pr}\left[|X|>(n \log \log n)^{\frac{1}{2}}\right]+\sum_{k=n}^{\infty} \int_{[k \log \log k]^{\frac{1}{2}}}^{[(k+1) \log \log (k+1)]^{\frac{1}{2}}} \operatorname{Pr}(|X|>x) d x \\
& \leqq(n \log \log n)^{\frac{1}{2}} \operatorname{Pr}\left[|X|>(n \log \log n)^{\frac{1}{2}}\right] \\
& +\sum_{k=n}^{\infty} \operatorname{Pr}\left[|X|>(k \log \log k)^{\frac{1}{2}}\right]\left\{[(k+1) \log \log (k+1)]^{\frac{1}{2}}-[k \log \log k]^{\frac{1}{2}}\right\} \\
& \leqq(n \log \log n)^{\frac{1}{2}} \operatorname{Pr}\left[|X|>(n \log \log n)^{\frac{1}{2}}\right] \\
& +C \sum_{k=n}^{\infty} k^{-\frac{1}{2}}(\log \log k)^{\frac{1}{2}} \operatorname{Pr}\left[|X|>(k \log \log k)^{\frac{1}{2}}\right] \\
& \leqq(n \log \log n)^{\frac{1}{2}} \operatorname{Pr}\left[|X|>(n \log \log n)^{\frac{1}{2}}\right] \\
& +C^{\prime} n^{-\frac{1}{2}}(\log \log n)^{\frac{1}{2}} \sum_{k=n}^{\infty} \operatorname{Pr}\left[|X|>(k \log \log k)^{\frac{1}{2}}\right] \text {, }
\end{aligned}
$$

$C, C^{\prime}$ being suitably chosen positive constants. Since $\sum \operatorname{Pr}\left[|X|>(n \log \log n)^{\frac{1}{2}}\right]$ $<\infty$, it follows immediately that (ii) is satisfied. We therefore have $(n \log \log n)^{-\frac{1}{2}} S_{n} \stackrel{P}{\rightarrow} 0$ and consequently that $(n \log \log n)^{-\frac{1}{2}} T_{n} \stackrel{P}{\rightarrow} 0$.

Next we return to the main problem. Define events $B_{n}$ by

$$
B_{n}=\left\{\left|U_{k(n)}\right| \geqq(N+\varepsilon)\left[(1+\eta) 2 b^{-1} k(n) \log \log k(n)\right]^{\frac{1}{2}}\right\} .
$$

Then from (1), our problem is to show that $\operatorname{Pr}\left(A_{n} \cap B_{n}\right.$ i.o. $)=1$, or equivalently, that $\lim _{n \rightarrow \infty} \operatorname{Pr}\left[\bigcup_{m=n}^{\infty}\left(A_{m} \cap B_{m}\right)\right]=1$.

Let $\bar{A}$ denote the complement of $A$. We have

$$
\begin{aligned}
\operatorname{Pr}\left[\bigcup_{m=n}^{\infty}\left(A_{m} \cap B_{m}\right)\right] & =\operatorname{Pr}\left[\bigcup_{m=n}^{\infty}\left\{\bigcap_{l=n}^{m-1} \overline{\left(A_{l} \cap B_{l}\right)} \cap\left(A_{m} \cap B_{m}\right)\right\}\right] \\
& =\sum_{m=n}^{\infty} \operatorname{Pr}\left[\bigcap_{l=n}^{m-1} \overline{\left(A_{l} \cap B_{l}\right)} \cap\left(A_{m} \cap B_{m}\right)\right]
\end{aligned}
$$




$$
\begin{aligned}
& \geqq \sum_{m=n}^{\infty} \operatorname{Pr}\left[\bigcap_{l=n}^{m-1} \bar{B}_{l} \cap B_{m} \cap A_{m}\right] \\
& =\sum_{m=n}^{\infty} \sum_{i=1}^{\frac{1}{2}(a+1) m} \operatorname{Pr}\left[\bigcap_{l=n}^{m-1} \bar{B}_{l} \cap B_{m} \cap A_{m} \cap\{j(m)=i\}\right] \\
& \geqq \sum_{m=n}^{\infty} \sum_{i=\delta m}^{\frac{1}{2}(a+1) m} \operatorname{Pr}\left[\bigcap_{l=n}^{m-1} \bar{B}_{l} \cap B_{m} \cap\{j(m)=i\}\right] \operatorname{Pr}\left[A_{m} \cap\{j(m)=i\}\right],
\end{aligned}
$$

for arbitrary $\delta, 0<\delta<a$, the $\left\{Y_{i}\right\}$ and $\left\{Z_{i}\right\}$ being mutually independent. Now, since $(n \log \log n)^{-\frac{1}{2}} T_{n} \stackrel{P}{\rightarrow} 0, \operatorname{Pr}\left[\left|T_{n}\right| \leqq \varepsilon(n \log \log n)^{\frac{1}{2}}\right] \rightarrow 1$ as $n \rightarrow \infty$. Thus, we can find an integer $I$ and a constant $K>0$ such that

$$
\operatorname{Pr}\left[A_{m} \cap\{j(m)=i\}\right] \geqq K \quad \text { for all } m \geqq n \geqq I, \delta m \leqq i \leqq \frac{1}{2}(a+1) m .
$$

Then, for $n \geqq I$,

$$
\begin{aligned}
\operatorname{Pr}\left[\bigcup_{m=n}^{\infty}\left(A_{m} \cap B_{m}\right)\right] & \geqq K \sum_{m=n}^{\infty} \sum_{i=\delta m}^{\frac{1}{2}(a+1) m} \operatorname{Pr}\left[\bigcap_{l=n}^{m-1} \bar{B}_{l} \cap B_{m} \cap\{j(m)=i\}\right] \\
& =K \sum_{m=n}^{\infty} \operatorname{Pr}\left[\bigcap_{l=n}^{m-1} \bar{B}_{l} \cap B_{m} \cap\left\{\delta m \leqq j(m) \leqq \frac{1}{2}(a+1) m\right\}\right] \\
\geqq & K \sum_{m=n}^{\infty} \operatorname{Pr}\left[\bigcap_{l=n}^{m-1} \bar{B}_{l} \cap B_{m}\right]-K \sum_{m=n}^{\infty} \operatorname{Pr}\left[\overline{\left\{m \leqq j(m) \leqq \frac{1}{2}(a+1) m\right\}}\right] .
\end{aligned}
$$

Now$$
\operatorname{Pr}\left[\overline{\left\{\delta m \leqq j(m) \leqq \frac{1}{2}(a+1) m\right\}}\right]=\operatorname{Pr}[j(m)<\delta m]+\operatorname{Pr}\left[j(m)>\frac{1}{2}(a+1) m\right]
$$$$
=\operatorname{Pr}[k(m)>(1-\delta) m]+\operatorname{Pr}\left[j(m)>\frac{1}{2}(a+1) m\right]
$$$$
\leqq e^{-(1-\delta) m t_{1}} E\left[e^{t_{1} k(m)}\right]+e^{-\frac{1}{2}(a+1) m t_{2}} E\left[e^{t_{2} j(m)}\right]
$$$$
=\left[e^{-t_{1}(1-\delta)}\left(a e^{t_{1}}+b\right)\right]^{m}+\left[e^{-\frac{1}{2}(a+1) t_{2}}\left(a-b e^{t_{2}}\right)\right]^{m}
$$$$
=\left[\xi\left(t_{1}\right)\right]^{m}+\left[\eta\left(t_{2}\right)\right]^{m},
$$

say, where $t_{1}$ and $t_{2}$ are positive and chosen so small that $\xi\left(t_{1}\right)<1, \eta\left(t_{2}\right)<1$. Then,

$$
\begin{aligned}
\sum_{m=n}^{\infty} \operatorname{Pr}\left[\overline{\left\{\delta m \leqq j(m) \leqq \frac{1}{2}(a+1) m\right\}}\right] & \leqq \frac{\left[\xi\left(t_{1}\right)\right]^{n}}{1-\xi\left(t_{1}\right)}+\frac{\left[\eta\left(t_{2}\right)\right]^{m}}{1-\eta\left(t_{2}\right)} \\
& \rightarrow 0 \text { as } n \rightarrow \infty
\end{aligned}
$$

Consequently, from (2),

$$
\begin{aligned}
\lim _{n \rightarrow \infty} \operatorname{Pr}\left[\bigcup_{m=n}^{\infty}\left(A_{m} \cap B_{m}\right)\right] & \geqq K \lim _{n \rightarrow \infty} \sum_{m=n}^{\infty} \operatorname{Pr}\left[\bigcup_{l=n}^{m-1}\left(\bar{B}_{l} \cap B_{m}\right)\right] \\
& =K \lim _{n \rightarrow \infty} \operatorname{Pr}\left[\bigcup_{m=n}^{\infty} B_{n}\right]=K
\end{aligned}
$$


for $(N+\varepsilon)^{2}(1+\eta)<\sigma b$ since $\operatorname{Pr}\left(B_{n}\right.$ i.o. $)=1$ under this condition. It then follows, from the zero-one law, that $\operatorname{Pr}\left(\left|S_{n}\right|>N(n \log \log n)^{\frac{1}{2}}\right.$ i.o. $)=1$ provided that $(N+\varepsilon)^{2}(1+\eta)<\sigma b$. However, $E X^{2}=\infty$, so $\sigma$ can be made arbitrarily large by increasing $K$ and, correspondingly, $b$ tends towards unity. $\varepsilon$ and $\eta$ are arbitrarily small so in fact the relation $\operatorname{Pr}\left(\left|S_{n}\right|>N(n \log \log n)^{\frac{1}{2}}\right.$ i.o. $)=1$ holds no matter how large be $N>0$. This establishes the result of the theorem.

\section{References}

[1] Hartman, P. And Wintner, A. (1941) On the law of the iterated logarithm. Amer. J. Math. 63, 169-176.

[2] LoÈve, M. (1963) Probability Theory. 3rd edition. Van Nostrand, New York. 1041.

[3] Stone, C. (1966) The growth of a recurrent random walk. Ann. Math. Statist. 37, 1040

[4] Strassen, V. (1966) A converse to the law of the iterated logarithm. Z. Wahrscheinlichkeitsth. 4, 265-268. 